\title{
Cerebral venous thrombosis
}

\author{
Emerson L. Gasparetto \\ Adjunct Professor of Radiology at Universidade Federal \\ do Rio de Janeiro, MD, Radiologist at DAPI (Curitiba) \\ and CDPI (Rio de Janeiro), Brazil.
}

Cerebral venous thrombosis (CVT) was described 150 years ago and still is considered as a severe condition. With an incidence of $0.1 \%$ in 12,500 consecutive autopsies, the CVT presents high mortality rates, ranging between $4 \%$ and $50 \%$ in most studies. The clinical presentation varies according to the portion of the cerebral venous system affected, and may start with headache of variable intensity and signs of increase in the intracranial pressure or through cerebral venous infarcts which frequently may be of hemorrhagic nature, causing seizures, neurological deficit and alterations of consciousness ${ }^{(1)}$.

Generally, cranial computed tomography (CT) is the first imaging examination performed in patients while still in the emergency department. In some cases, CT may demonstrate an increased density of thrombosed venous sinuses which may cause the empty delta sign on contrast-enhanced images. However, in the majority of cases, the findings are non-specific and include hypodensities, hemorrhages, contrast uptake. Additionally, about $30 \%$ of patients with CVT present normal cranial CT scans at the beginning of the clinical picture ${ }^{(1,2)}$.

Encephalic magnetic resonance imaging (MRI) is the gold standard for CVT diagnosis, the conventional angiography being reserved for cases of cortical veins involvement or with doubtful findings at MRI. Usually, MRI demonstrates the thrombosed sinus with high-intensity signal on T1- and T2-weighted images. These signal characteristics may vary according to the time elapsed between the CVT onset and the magnetic resonance images acquisition. Besides, mass effect lesions are frequently seen in patients with
CVT, many times associated with areas of hypersignal on T2-weighted images of the cerebral parenchyma, possibly involving the cortex, the white matter, or both, and intraparenchymal hematomas ${ }^{(2,3)}$.

In the interesting study of Ferreira et al., included in this issue of Radiologia Brasileira, MRI and MRI angiography of 21 patients with CVT have been studied, emphasizing the parenchymal abnormalities in this group of patients. The main findings were: areas of cortical or subcortical edema/infarct, venous congestion, meningeal enhancement and basal ganglia edema/infarct. The main venous sinuses affected were the superior sagittal, the left transverse, the left sigmoid and the straight sinuses. The majority of these findings corroborate the already published literature on this matter.

In summary, CVT diagnosis is difficult and frequently its clinical presentation may mimic other diseases, causing irreparable injury to the patient, because of the delayed diagnosis. Many times, imaging findings are not so clear in cases of patients with CVT and this diagnostic hypothesis should ever be taken into consideration.

\section{REFERENCES}

1. Ehtisham A, Stern BJ. Cerebral venous thrombosis: a review. Neurologist 2006;12:32-38.

2. Provenzale JM, Joseph GJ, Barboriak DP. Dural sinus thrombosis: findings on CT and MR imaging and diagnostic pitfalls. AJR Am J Roentgenol 1998;170:777-783.

3. Mullins ME, Grant PE, Wang B, Gonzalez RG, Schaefer PW. Parenchymal abnormalities associated with cerebral venous sinus thrombosis: assessment with diffusion-weighted MR imaging. AJNR Am J Neuroradiol 2004;25:1666-1675. 